Research Article

\title{
Biharmonic Hypersurfaces in Pseudo-Riemannian Space Forms with at Most Two Distinct Principal Curvatures
}

\author{
Chao Yang $(\mathbb{D}$ and Jiancheng Liu \\ College of Mathematics and Statistics, Northwest Normal University, Lanzhou 730070, China \\ Correspondence should be addressed to Chao Yang; yc963852@126.com
}

Received 18 May 2020; Accepted 6 July 2020; Published 1 August 2020

Academic Editor: Raúl E. Curto

Copyright (c) 2020 Chao Yang and Jiancheng Liu. This is an open access article distributed under the Creative Commons Attribution License, which permits unrestricted use, distribution, and reproduction in any medium, provided the original work is properly cited.

In this paper, we show that biharmonic hypersurfaces with at most two distinct principal curvatures in pseudo-Riemannian space form $N_{s}^{n+1}(c)$ with constant sectional curvature $c$ and index $s$ have constant mean curvature. Furthermore, we find that such biharmonic hypersurfaces $M_{r}^{2 k-1}$ in even-dimensional pseudo-Euclidean space $\mathbb{E}_{s}^{2 k}, M_{s-1}^{2 k-1}$ in even-dimensional de Sitter space $\mathbb{S}_{s}^{2 k}(c)(c>0)$, and $M_{s}^{2 k-1}$ in even-dimensional anti-de Sitter space $\mathbb{H}_{s}^{2 k}(c)(c<0)$ are minimal.

\section{Introduction}

Let $N_{s}^{n+1}(c)$ be a $(n+1)$-dimensional pseudo-Riemannian space form with index $1 \leq s \leq n+1$ and constant sectional curvature $c$. According to $c=0, c>0$, and $c<0, N_{s}^{n+1}(c)$ is isometric to pseudo-Euclidean space $\mathbb{E}_{s}^{n+1}$, de Sitter space $\mathbb{S}_{s}^{n+1}(c)$, and anti-de Sitter space $\mathbb{H}_{s}^{n+1}(c)$.

Suppose that $\phi: M_{r}^{n} \rightarrow N_{s}^{n+1}(c), r=s-1$, or $s$ be an isometric immersion of a pseudo-Riemannian hypersurface $M_{r}^{n}$ into $N_{s}^{n+1}(c)$. The hypersurface $M_{r}^{n}$ is said to be biharmonic if its bitension field $\tau_{2}(\phi)$ vanishes identically, i.e.,

$$
\tau_{2}(\phi):=\operatorname{trace}\left(\nabla^{\phi} \nabla^{\phi}-\nabla_{\nabla}^{\phi}\right) \tau(\phi)-\operatorname{trace} \tilde{R}(\mathrm{~d} \phi, \tau(\phi)) \mathrm{d} \phi=0,
$$

where $\tau(\phi):=\operatorname{div}(\mathrm{d} \phi), \tilde{R}, \nabla^{\phi}$, and $\nabla$ are the curvature tensor of $N_{s}^{n+1}(c)$, the induced connection by $\phi$ on the bundle $\phi^{-1}$ $T N_{s}^{n+1}(c)$, and the connection of $M_{r}^{n}$, respectively. If the mean curvature of the hypersurface $M_{r}^{n}$ is zero, then we call $M_{r}^{n}$ as minimal. It is generally known that minimal hypersurfaces are biharmonic ones. Conversely, the natural question is whether any biharmonic hypersurface is minimal.
For biharmonic hypersurfaces in pseudo-Euclidean spaces, there is a conjecture in [2] that every biharmonic hypersurface of pseudo-Euclidean space $\mathbb{E}_{s}^{n+1}$ is minimal. Up to now, this conjecture has been examined for many biharmonic hypersurfaces, such as $M_{r}^{2}$ of $\mathbb{E}_{s}^{3}$ (cf. [2, 3]), $M_{1}^{3}$ of $\mathbb{E}_{1}^{4}$ (cf. [1]), $M_{2}^{3}$ of $\mathbb{E}_{2}^{4}$ (cf. [10]), and $M_{r}^{n}$ in $\mathbb{E}_{s}^{n+1}$ with at most three distinct principal curvatures and diagonalizable shape operator (cf. $[5,7])$.

When the ambient space is de Sitter space $\mathbb{S}_{s}^{n+1}(c)$, there are also some papers that studied the above problem. Sasahara in [11] considered biharmonic hypersurfaces $M_{r}^{2}$ of $\mathbb{S}_{1}^{3}(c)$ and proved that it must be minimal when $r=0$, but may not when $r=1$. Investigators studied biharmonic hypersurfaces with at most two distinct principal curvatures in $\mathbb{S}_{s}^{n+1}(c)$ whose shape operator is diagonalizable in $[6,8]$ and showed that such hypersurface $M_{s-1}^{n}$ is minimal, but the hypersurface $M_{s}^{n}$ may not. Naturally, there is a question as to whether any biharmonic hypersurface $M_{s-1}^{n}$ in de Sitter space $\mathbb{S}_{s}^{n+1}(c)$ is minimal.

The situation is quite different when the ambient space is anti-de Sitter space $\mathbb{H}_{s}^{n+1}(c)$. For biharmonic hypersurface $M_{r}^{2}$ of $\mathbb{H}_{1}^{3}(c)$, it must be minimal when $r=1$ and may not when $r=0$ (cf. [11]). For biharmonic hypersurface $M_{r}^{n}$ with at most two distinct principal curvatures in $\mathrm{H}_{s}^{n+1}(c)$ whose shape operator is diagonalizable, it is minimal when $r=s$ 
and may not when $r=s-1$ (cf. $[6,8]$ ). A natural question is whether any biharmonic hypersurface $M_{s}^{n}$ in anti-de Sitter space $\mathbb{H}_{s}^{n+1}(c)$ is minimal.

In this paper, we study biharmonic hypersurfaces with at most two distinct principal curvatures in pseudo-Riemannian space forms $N_{s}^{n+1}(c)$, without the restriction that the shape operator is diagonalizable. We proved such biharmonic hypersurfaces have constant mean curvature. Furthermore, we find that such biharmonic hypersurfaces $M_{r}^{2 k-1}$ in even-dimensional pseudo-Euclidean space $\mathbb{E}_{s}^{2 k}$, $M_{s-1}^{2 k-1}$ in even-dimensional de Sitter space $\mathbb{S}_{s}^{2 k}(c)$, and $M_{s}^{2 k-1}$ in even-dimensional anti-de Sitter space $\mathbb{H}_{s}^{2 k}(c)$ are minimal.

\section{Preliminaries}

2.1. Notions and Formulas of Hypersurfaces in $N_{s}^{n+1}(c)$. Let $N_{s}^{n+1}(c)$ be a pseudo-Riemannian space form with index $s$ and constant sectional curvature $c$. A nonzero vector $X$ in $N_{s}^{n+1}(c)$ is called time-like, space-like, or light-like, according to whether $\langle X, X\rangle$ is negative, positive, or zero, respectively. Let $M_{r}^{n}$ be a nondegenerate hypersurface in $N_{s}^{n+1}(c)$. $\vec{\xi}$ denotes a unit normal vector field to $M_{r}^{n}$, then $\varepsilon=\langle\vec{\xi}, \vec{\xi}\rangle= \pm$ 1. Denote by $\nabla$ and $\tilde{\nabla}$ the Levi-Civita connections of $M_{r}^{n}$ and $N_{s}^{n+1}(c)$, respectively. For any vector fields $X, Y$ tangent to $M_{r}^{n}$, the Gauss formula and Weingarten formula are given by

$$
\begin{aligned}
& \tilde{\nabla}_{X} Y=\nabla_{X} Y+h(X, Y) \vec{\xi}, \\
& \tilde{\nabla}_{X} \vec{\xi}=-A(X),
\end{aligned}
$$

where $h$ is the scalar-valued second fundamental form and $A$ is the shape operator of $M_{r}^{n}$ associated to $\vec{\xi}$. The mean curvature vector field $H$ can be expressed as $\vec{H}=H \vec{\xi}$, with mean curvature $H=(1 / n) \varepsilon \operatorname{tr} A$. For any vector fields $X, Y, Z$ tangent to $M_{r}^{n}$, the Codazzi and Gauss equations are (cf. [9])

$$
\begin{aligned}
\left(\nabla_{X} A\right) Y= & \left(\nabla_{Y} A\right) X \\
R(X, Y) Z= & c(\langle Y, Z\rangle X-\langle X, Z\rangle Y)+\varepsilon\langle A(Y), Z\rangle A(X) \\
& -\varepsilon\langle A(X), Z\rangle A(Y),
\end{aligned}
$$

here $R(X, Y) Z=\nabla_{X} \nabla_{Y} Z-\nabla_{Y} \nabla_{X} Z-\nabla_{[X, Y]} Z$.

A hypersurface $M_{r}^{n}$ of $N_{s}^{n+1}(c)$ is biharmonic if and only if its mean curvature $H$ satisfies the following two equations (cf. [4]):

$$
\begin{aligned}
A(\nabla H) & =-\frac{n}{2} \varepsilon H(\nabla H), \\
\Delta H+\varepsilon H \operatorname{tr} A^{2} & =n c H,
\end{aligned}
$$

where

$$
\Delta H=-\sum_{i=1}^{n}\left(e_{i} e_{i} H-\nabla_{e_{i}} e_{i} H\right),
$$

here $\left\{e_{1}, e_{2}, \cdots, e_{n}\right\}$ is a local orthonormal frame of $T_{x} M_{r}^{n}$.

2.2. The Shape Operator of $M_{r}^{n}$ in $N_{s}^{n+1}(c)$. According to [9] (exercise 18, pp. 260-261), the tangent space $T_{x} M_{r}^{n}$ at $x \in$ $M_{r}^{n}$ can be expressed as a direct sum of subspaces $V_{k}, 1 \leq k$ $\leq m$, that are mutually orthogonal and invariant under the shape operator $A$, and each $\left.A\right|_{V_{k}}$ (the restriction of $A$ on $V_{k}$ ) has form (a) or (b) as follows.

(a) $\left.A\right|_{V_{k}}$ has the form

$$
\left.A\right|_{V_{k}}=\left(\begin{array}{ccccc}
\lambda_{k} & & & & \\
1 & \lambda_{k} & & & \\
& \ddots & \ddots & & \\
& & 1 & \lambda_{k} & \\
& & & 1 & \lambda_{k}
\end{array}\right) \text {, }
$$

with respect to a basis $\mathfrak{B}_{k}=\left\{u_{k_{1}}, u_{k_{2}}, \cdots, u_{k_{\alpha_{k}}}\right\}$ of $V_{k}$. The inner products of the basis elements in $\mathfrak{B}_{k}$ are all zero except

$$
\left\langle u_{k_{b}}, u_{k_{d}}\right\rangle=\varepsilon_{k}= \pm 1, \quad b+d=\alpha_{k}+1
$$

(b) $\left.A\right|_{V_{k}}$ has the form

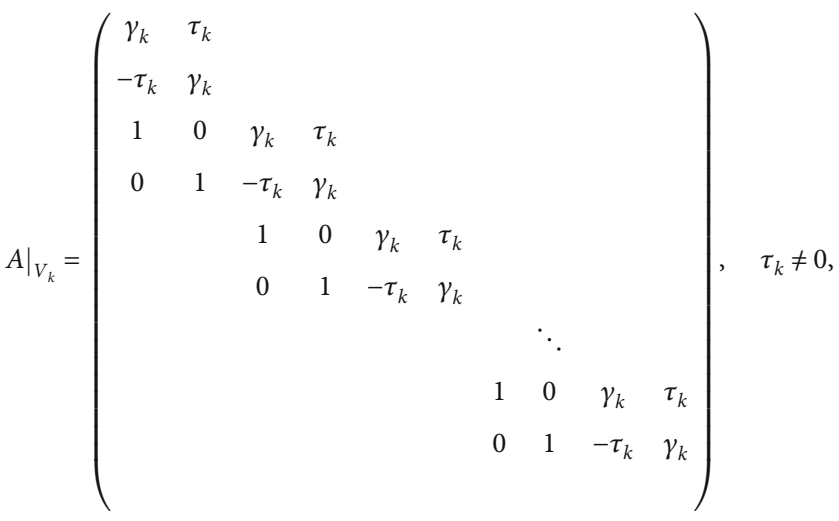

with respect to a basis $\overline{\mathfrak{B}}_{k}=\left\{\bar{u}_{1_{1}}, \bar{v}_{1_{1}}, \cdots, \bar{u}_{k_{\beta_{k}}}, \bar{v}_{k_{\beta_{k}}}\right\}$ of $V_{k}$. The inner products of the basis elements in $\overline{\mathfrak{B}}_{k}$ are all zero except

$$
\left\langle\bar{u}_{k_{b}}, \bar{u}_{k_{d}}\right\rangle=1=-\left\langle\bar{v}_{k_{b}}, \bar{v}_{k_{d}}\right\rangle, \quad b+d=\beta_{k}+1 .
$$

We denote by $t$ the number of terms $\left.A\right|_{V_{k}}$ having form (a). We adjust the order of $V_{k}, 1 \leq k \leq m$, such that $\left.A\right|_{V_{k}}$ have 
form (a) for $1 \leq k \leq t$ and $\left.A\right|_{V_{k}}$ have form (b) for $t+1 \leq k \leq m$. Denote $A_{i}=\left.A\right|_{V_{i}}, 1 \leq i \leq t$, and $\bar{A}_{j}=\left.A\right|_{V_{j}}, t+1 \leq j \leq m$. Collecting all the vectors in $\mathfrak{B}_{1}, \cdots, \mathfrak{B}_{t}, \overline{\mathfrak{B}}_{t+1}, \cdots, \overline{\mathfrak{B}}_{m}$ in order, we get a basis $\mathfrak{B}$ of $T_{x} M_{r}^{n}$. With respect to this basis $\mathfrak{B}$, the shape operator $A$ of the hypersurface $M_{r}^{n}$ in $N_{s}^{n+1}(c)$ can be expressed as an almost diagonal matrix:

$$
A=\operatorname{diag}\left\{A_{1}, \cdots, A_{t}, \bar{A}_{t+1}, \cdots, \bar{A}_{m}\right\},
$$

and the inner products of the elements in $\mathfrak{B}$ are all zero except

$$
\begin{aligned}
\left\langle u_{i_{a}}, u_{i_{b}}\right\rangle & =\varepsilon_{i}= \pm 1, a+b=\alpha_{i}+1,1 \leq i \leq t \\
\left\langle\bar{u}_{j_{c}}, \bar{u}_{j_{d}}\right\rangle & =1=-\left\langle\bar{v}_{j_{c}}, \bar{v}_{j_{d}}\right\rangle, c+d=\beta_{j}+1, t+1 \leq j \leq m,
\end{aligned}
$$

where

$$
\alpha_{1}+\alpha_{2}+\cdots+\alpha_{t}+2\left(\beta_{t+1}+\beta_{t+2}+\cdots+\beta_{m}\right)=n .
$$

Observe the forms (a) and (b); we see that $A_{i}, 1 \leq i \leq t$, has only a simple eigenvalue $\lambda_{i}$ and $\bar{A}_{j}, t+1 \leq j \leq m$, has eigenvalues $\gamma_{j}+\tau_{j} \sqrt{-1}, \gamma_{j}-\tau_{j} \sqrt{-1}$. It follows from the form of the shape operator $A$ that $M_{r}^{n}$ has principal curvatures

$$
\lambda_{1}, \cdots, \lambda_{t}, \quad \gamma_{t+1} \pm \tau_{t+1} \sqrt{-1}, \cdots, \gamma_{m} \pm \tau_{m} \sqrt{-1}
$$

So, under the assumption that $M_{r}^{n}$ has at most two distinct principal curvatures, the shape operator $A$ has the following two possible forms:

(I) $t=m$, i.e., $A=\operatorname{diag}\left\{A_{1}, A_{2}, \cdots, A_{m}\right\}$, and there are at most two distinct values among $\left\{\lambda_{1}, \lambda_{2}, \cdots, \lambda_{m}\right\}$

(II) $t=0$, i.e., $A=\operatorname{diag}\left\{\bar{A}_{1}, \bar{A}_{2}, \cdots, \bar{A}_{m}\right\}$ and $\gamma_{1}=\gamma_{2}=\cdots$ $=\gamma_{m}=\gamma, \tau_{1}=\tau_{2}=\cdots=\tau_{m}=\tau, \tau \neq 0$

For the form (I), we have

$$
A\left(u_{i_{a}}\right)=\lambda_{i} u_{i_{a}}+u_{i_{a+1}}, A\left(u_{i_{\alpha_{i}}}\right)=\lambda_{i} u_{i_{\alpha_{i}}}, \quad 1 \leq i \leq m, 1 \leq a \leq \alpha_{i}-1 .
$$

And for the form (II), we have

$$
\begin{aligned}
& A\left(\bar{u}_{j_{b}}\right)=v \bar{u}_{j_{b}}-\tau \bar{v}_{j_{b}}+\bar{u}_{j_{b+1}}, A\left(\bar{u}_{j_{\beta_{j}}}\right)=v \bar{u}_{j_{\beta_{j}}}-\tau \bar{v}_{j_{\beta_{j}}}, \\
& A\left(\bar{v}_{j_{b}}\right)=\tau \bar{u}_{j_{b}}+v \bar{v}_{j_{b}}+\bar{v}_{j_{b+1}}, A\left(\bar{v}_{j_{\beta_{j}}}\right)=\tau \bar{u}_{j_{\beta_{j}}}+v \bar{v}_{j_{\beta_{j}}},
\end{aligned}
$$

with $t+1 \leq j \leq m, 1 \leq b \leq \beta_{j}$.

\section{Theorems}

Theorem 1. Let $N_{s}^{n+1}(c)$ be a $(n+1)$-dimensional pseudoRiemannian space form with index $s$ and constant sectional curvature $c$ and $M_{r}^{n}$ be a nondegenerate biharmonic hypersurface of $N_{s}^{n+1}(c)$ with at most two distinct principal curvatures, then $M_{r}^{n}$ has constant mean curvature.

Proof. From Section 2, the shape operator $A$ has the form (I) or (II). If $A$ has the form (II), then its eigenvalues are not real and $-(n / 2) \varepsilon H$ is not an eigenvalue. It follows from (5) that $\nabla H=0$, which tells us $H$ is a constant.

For the form (I), if we assume that $H$ is not a constant, then (5) implies that $-(n / 2) \varepsilon H$ is an eigenvalue of the shape operator $A$. When $\lambda_{1}=\cdots=\lambda_{m}$, then $\operatorname{tr} A=-\left(n^{2} / 2\right) \varepsilon H$. On the other hand, $\operatorname{tr} A=n \varepsilon H$. These two expressions imply $H=0$, a contradiction.

So, in the following, we need only to discuss the situation where there are two distinct values among $\left\{\lambda_{1}, \cdots, \lambda_{m}\right\}$. Expression (5) also informs us that $\nabla H$ is an eigenvector of $A$ with corresponding eigenvalue $-(n / 2) \varepsilon H$. In view of (16), $\nabla H$ is one of the directions $u_{i_{\alpha_{i}}}, 1 \leq i \leq m$. Without loss of generality, we suppose $\nabla H$ is in the direction of $u_{1_{\alpha_{1}}}$; it may be a light-like vector or not. We will follow different processes to lead contradictions for these two cases.

First of all, we give a lot of equations deduced from compatibility and symmetry of the connection, as well as the Codazzi equation.

Observe the inner products of the elements in basis $\mathfrak{B}$ given in Section 2, we can express

$$
\nabla H=\sum_{i=1}^{m} \sum_{a=1}^{\alpha_{i}} \varepsilon_{i} u_{i_{\alpha_{i}-a+1}}(H) u_{i_{a}} .
$$

Since $\nabla H$ is in the direction of $u_{1_{\alpha_{1}}}$, the above equation implies that

$$
u_{1_{1}}(H) \neq 0, u_{i_{a}}(H)=0, \quad i_{a} \neq 1_{1} .
$$

Let $\nabla_{u_{i_{a}}} u_{j_{b}}=\sum_{k=1}^{m} \sum_{d=1}^{\alpha_{k}} \Gamma_{i_{a} j_{b}}^{k_{d}} u_{k_{d}}$. Applying compatibility condition to calculate

$$
\nabla_{u_{D}}\left\langle u_{i_{a}}, u_{i_{a}}\right\rangle, \nabla_{u_{D}}\left\langle u_{i_{a}}, u_{i_{b}}\right\rangle, \nabla_{u_{D}}\left\langle u_{i_{a}}, u_{j_{d}}\right\rangle
$$

we conclude

$$
\begin{aligned}
& \Gamma_{D i_{a}}^{i_{\alpha_{i}-a+1}}=0, \\
& \Gamma_{D i_{a}}^{i_{\alpha_{i}-b+1}}=-\Gamma_{D i_{b}}^{i_{\alpha_{i}-a+1}}, \Gamma_{D i_{a}}^{j_{\alpha_{j}-d+1}}=-\varepsilon_{i} \varepsilon_{j} \Gamma_{D j_{d}}^{i_{\alpha_{i}-a+1}},
\end{aligned}
$$

for $D \in\left\{k_{e}, 1 \leq k \leq m, 1 \leq e \leq \alpha_{k}\right\}, 1 \leq i, j \leq m, 1 \leq a, b \leq \alpha_{i}$, and $1 \leq d \leq \alpha_{j}$.

From the expression $\left(\nabla_{u_{B}} u_{C}-\nabla_{u_{C}} u_{B}\right)(H)=\left[u_{B}, u_{C}\right](H)$, $B, C \in\left\{k_{d}, 1 \leq k \leq m, 1 \leq d \leq \alpha_{k}\right\}$, and (19), we easily get

$$
\Gamma_{B C}^{1_{1}}=\Gamma_{C B}^{1_{1}}, \quad B, C \neq 1_{1} .
$$

We state that in the proof, if not otherwise specified, then for $i_{a}$ in $u_{i_{a}}$ and the connection's coefficients, the ranges of $i$ 
and $a$ are as follows: $1 \leq i \leq m$ and $1 \leq a \leq \alpha_{i}$. For the equations about the connection's coefficients, when $a=\alpha_{i}$ (or $a=$ 1 ), then the terms about $i_{a+1}$ (or $i_{a-1}$ ) disappear. And when $b=\beta_{j}($ or $b=1)$, then the terms about $\bar{j}_{b+1}\left(\right.$ or $\left.\bar{j}_{b-1}\right)$ and $\tilde{j}_{b+1}$ (or $\tilde{j}_{b-1}$ ) disappear.

It follows from the Codazzi equation (3) that for any vector fields $X, Y, Z$ tangent to $M_{r}^{n}$,

$$
\left\langle\left(\nabla_{X} A\right) Y, Z\right\rangle=\left\langle\left(\nabla_{Y} A\right) X, Z\right\rangle
$$

Start with this equation, we can get a series of equations about the coefficients of connection.

(i) For $(X, Y, Z)=\left(u_{1_{a}}, u_{1_{b}}, u_{1_{\alpha_{1}}}\right)$ in (24), then combining (19), we obtain

$$
\Gamma_{1_{a} 1_{b+1}}^{1_{1}}=\Gamma_{1_{b} 1_{a+1}}^{1_{1}}
$$

Applying (21), (22), and (23), we get from the above equation that

$$
\Gamma_{1_{\alpha_{1}-1} 1_{\alpha_{1}}}^{1_{\alpha_{1}-1}}=\cdots=\Gamma_{1_{2} 1_{\alpha_{1}}}^{1_{2}}=\Gamma_{1_{1} 1_{\alpha_{1}}}^{1_{1}}=0
$$

Note that if $\alpha_{1}=1$, then (26) tells us nothing.

(ii) For $(X, Y, Z)=\left(u_{1_{a}}, u_{i_{b}}, u_{1_{\alpha_{1}}}\right), 2 \leq i \leq m$, in (24), then combining (19), we have

$$
\left(\lambda_{i}+\frac{n}{2} \varepsilon H\right) \Gamma_{1_{a} i_{b}}^{1_{1}}+\Gamma_{1_{a} i_{b+1}}^{1_{1}}=\Gamma_{i_{b} 1_{a+1}}^{1_{1}}, \quad 2 \leq i \leq m
$$

which together with (23) implies that if $\lambda_{i}=-(n / 2) \varepsilon H$, then

$$
\Gamma_{1_{a+1} i_{\alpha_{i}}}^{1_{1}}=0
$$

and if $\lambda_{i} \neq-(n / 2) \varepsilon H$, then

$$
\Gamma_{1_{a} i_{b}}^{1_{1}}=0
$$

(iii) For $(X, Y, Z)=\left(u_{i_{a}}, u_{1_{\alpha_{1}}}, u_{1_{\alpha_{1}-b+1}}\right), 2 \leq i \leq m$, in (24), then

$$
-\Gamma_{i_{a} 1_{\alpha_{1}}}^{1_{b-1}}=\left(\lambda_{i}+\frac{n \varepsilon H}{2}\right) \Gamma_{1_{\alpha_{1}} i_{a}}^{1_{b}}+\Gamma_{1_{\alpha_{1}} i_{a+1}}^{1_{b}}-\Gamma_{1_{\alpha_{1}} i_{a}}^{1_{b-1}},
$$

which together with (22), (23), (28), and (29) implies that if $\lambda_{i} \neq-(n / 2) \varepsilon H$, then

$$
\Gamma_{1_{\alpha_{1}} i_{a}}^{1_{b}}=0, \quad 2 \leq i \leq m .
$$

(iv) For $(X, Y, Z)=\left(u_{i_{a}}, u_{i_{b}}, u_{1_{\alpha_{1}}}\right), 2 \leq i \leq m$, in (24), then combining (23), we know

$$
\Gamma_{i_{a} i_{b+1}}^{1_{1}}=\Gamma_{i_{b} i_{a+1}}^{1_{1}} .
$$

From (22), (23), and the above relation, we conclude that

$$
\Gamma_{i_{\alpha_{i}} i_{b+1}}^{1_{1}}=0, \quad 2 \leq i \leq m
$$

$$
\Gamma_{i_{a} 1_{\alpha_{1}}}^{i_{b}}=\Gamma_{i_{a+1} 1_{\alpha_{1}}}^{i_{b+1}}, \quad 2 \leq i \leq m, 1 \leq a, b \leq \alpha_{i}-1 .
$$

It follows from (33) and (34) that if $a>b$, then

$$
\Gamma_{i_{a} 1_{\alpha_{1}}}^{i_{b}}=0
$$

(v) For $(X, Y, Z)=\left(u_{i_{a}}, u_{1_{\alpha_{1}}}, u_{i_{\alpha_{i}-a+1}}\right), 2 \leq i \leq m$, in (24), then

$$
\left(-\frac{n}{2} \varepsilon H-\lambda_{i}\right) \Gamma_{i_{a} 1_{\alpha_{1}}}^{i_{a}}-\Gamma_{i_{a} 1_{\alpha_{1}}}^{i_{a-1}}=u_{1_{\alpha_{1}}}\left(\lambda_{i}\right)+\Gamma_{1_{\alpha_{1}} i_{a+1}}^{i_{a}}-\Gamma_{1_{\alpha_{1}} i_{a},}^{i_{a-1}},
$$

which together with (22) and (34) gives that

$$
\begin{gathered}
\Gamma_{1_{\alpha_{1}} i_{2}}^{i_{1}}=\Gamma_{1_{\alpha_{1}} i_{3}}^{i_{2}}=\cdots=\Gamma_{1_{\alpha_{1}} i_{\alpha_{i}}}^{i_{\alpha_{i}-1}}=0, \quad 2 \leq i \leq m, \\
u_{1_{\alpha_{1}}}\left(\lambda_{i}\right)=\left(-\frac{n}{2} \varepsilon H-\lambda_{i}\right) \Gamma_{i_{\alpha_{i}} 1_{\alpha_{1}}}^{i_{\alpha_{i}}}, \quad 2 \leq i \leq m .
\end{gathered}
$$

(vi) For $(X, Y, Z)=\left(u_{i_{a}}, u_{1_{\alpha_{1}}}, u_{i_{\alpha_{i}}}\right), 2 \leq i \leq m, 2 \leq a \leq \alpha_{i}$, in (24), then

$$
\left(-\frac{n}{2} \varepsilon H-\lambda_{i}\right) \Gamma_{i_{a} 1_{\alpha_{1}}}^{i_{1}}=\Gamma_{1_{\alpha_{1}} i_{a+1}}^{i_{1}}
$$

which combining (35) and (37), implies that

$$
\Gamma_{1_{\alpha_{1}} i_{b}}^{i_{1}}=0, \quad 2 \leq i \leq m, 2 \leq b \leq \alpha_{i} .
$$

(vii) For $(X, Y, Z)=\left(u_{i_{a}}, u_{j_{b}}, u_{1_{\alpha_{1}}}\right), 2 \leq i, j \leq m$, in (24), then combining (23), we have

$$
\left(\lambda_{j}-\lambda_{i}\right) \Gamma_{i_{a} j_{b}}^{1_{1}}+\Gamma_{i_{a} j_{b+1}}^{1_{1}}=\Gamma_{j_{b} i_{a+1}}^{1_{1}}
$$

which together with (23) tells us that when $\lambda_{i} \neq \lambda_{j}$, then

$$
\Gamma_{i_{a} j_{b}}^{1_{1}}=0, \quad 2 \leq i, j \leq m,
$$


and when $\lambda_{i}=\lambda_{j}$, then

$$
\begin{aligned}
& \Gamma_{i_{\alpha_{i}} j_{b+1}}^{1_{1}}=0, \\
& \Gamma_{i_{a} 1_{\alpha_{1}}}^{j_{b}}=\Gamma_{i_{a+1} 1_{\alpha_{1}}}^{j_{b+1}},
\end{aligned}
$$

with $2 \leq i, j \leq m, 1 \leq a \leq \alpha_{i}, 1 \leq b \leq \alpha_{j}-1$. (43) and (44) give that if $a>b$, then

$$
\Gamma_{i_{a} 1_{\alpha_{1}}}^{j_{b}}=0 .
$$

(viii) For $(X, Y, Z)=\left(u_{i_{a}}, u_{1_{\alpha_{1}}}, u_{j_{\alpha_{j}-b+1}}\right)$ with $2 \leq i, j \leq m$, and $i \neq j$ in (24), then

$\left(-\frac{n \varepsilon H}{2}-\lambda_{j}\right) \Gamma_{i_{a} 1_{\alpha_{1}}}^{j_{b}}-\Gamma_{i_{a} a_{\alpha_{1}}}^{j_{b-1}}=\left(\lambda_{i}-\lambda_{j}\right) \Gamma_{1_{\alpha_{1}} i_{a}}^{j_{b}}+\Gamma_{1_{\alpha_{1}} i_{a+1}}^{j_{b}}-\Gamma_{1_{\alpha_{1}} i_{a}}^{j_{b_{1}}}$.

(ix) For $(X, Y, Z)=\left(u_{i_{a}}, u_{j_{b}}, u_{i_{\alpha_{i}-d+1}}\right), a \neq d$, in (24), with $\lambda_{j}=-(n / 2) \varepsilon H$ and $\lambda_{i} \neq-(n / 2) \varepsilon H$, then

$$
\left(-\frac{n}{2} \varepsilon H-\lambda_{i}\right) \Gamma_{i_{a} j_{b}}^{i_{d}}+\Gamma_{i_{a} j_{b+1}}^{i_{d}}-\Gamma_{i_{a} j_{b}}^{i_{d-1}}=\Gamma_{j_{b} i_{a+1}}^{i_{d}}-\Gamma_{j_{b} i_{a}}^{i_{d-1}} .
$$

Let

$$
\left(\begin{array}{l}
A_{i, 1, j_{b}}=\Gamma_{i_{2} j_{b}}^{i_{1}}+\Gamma_{i_{3} j_{b}}^{i_{2}}+\cdots+\Gamma_{i_{\alpha_{i}} j_{b}}^{i_{\alpha_{i}}, 1} \\
A_{i, 2, j_{b}}=\Gamma_{i_{3} j_{b}}^{i_{1}}+\Gamma_{i_{4} j_{b}}^{i_{2}}+\cdots+\Gamma_{i_{\alpha_{i}} j_{b}}^{i_{\alpha_{j}-2}}, \\
\vdots \\
A_{i, \alpha_{i}-1, j_{b}}=\Gamma_{i_{\alpha_{i}} j_{b}}^{i_{1}},
\end{array}\right.
$$

with $1 \leq i, j \leq m, 1 \leq b \leq \alpha_{j}$ and $\lambda_{j}=-(n / 2) \varepsilon H, \lambda_{i} \neq-(n / 2) \varepsilon$ $H$. Since (47), we find

$$
\left(\begin{array}{l}
\left(-\frac{n}{2} \varepsilon H-\lambda_{i}\right) A_{i, e, j_{b}}+A_{i, e, j_{b+1}}-A_{i, e+1, j_{b}}=0, \quad 1 \leq b \leq \alpha_{j}, 1 \leq e \leq \alpha_{i}-2, \\
\left(-\frac{n}{2} \varepsilon H-\lambda_{i}\right) A_{i, \alpha_{i}-1, j_{b}}+A_{i, \alpha_{i}-1, j_{b+1}}=0, \quad 1 \leq b \leq \alpha_{j},
\end{array}\right.
$$

which implies that

$$
A_{i, e, j_{b}}=0, \quad 1 \leq b \leq \alpha_{j}, 1 \leq e \leq \alpha_{i}-1,
$$

with $\lambda_{j}=-(n / 2) \varepsilon H, \lambda_{i} \neq-(n / 2) \varepsilon H$, and $1 \leq i, j \leq m$.

(x) For $(X, Y, Z)=\left(u_{i_{a}}, u_{j_{b}}, u_{i_{\alpha_{i}-a+1}}\right)$ in (24), with $\lambda_{j}=-$ $(n / 2) \varepsilon H$ and $\lambda_{i} \neq-(n / 2) \varepsilon H$, then

$$
\left(-\frac{n}{2} \varepsilon H-\lambda_{i}\right) \Gamma_{i_{a} j_{b}}^{i_{a}}+\Gamma_{i_{a} j_{b+1}}^{i_{a}}-\Gamma_{i_{a} j_{b}}^{i_{a-1}}=u_{j_{b}}\left(\lambda_{i}\right)+\Gamma_{j_{b} i_{a+1}}^{i_{a}}-\Gamma_{j_{b} i_{a}}^{i_{a-1}}
$$

which combining (50) gives that

$$
\left(-\frac{n}{2} \varepsilon H-\lambda_{i}\right) \sum_{a=1}^{\alpha_{i}} \Gamma_{i_{a} j_{b}}^{i_{a}}+\sum_{a=1}^{\alpha_{i}} \Gamma_{i_{a} j_{b+1}}^{i_{a}}=\alpha_{i} u_{j_{b}}\left(\lambda_{i}\right) .
$$

(xi) For $(X, Y, Z)=\left(u_{i_{a}}, u_{j_{b}}, u_{k_{\alpha_{k}-d+1}}\right)$ in (24), with $\lambda_{j}=-$ $(n / 2) \varepsilon H, \lambda_{i}=\lambda_{k} \neq-(n / 2) \varepsilon H$, and $i \neq k$, then

$$
\left(-\frac{n}{2} \varepsilon H-\lambda_{k}\right) \Gamma_{i_{a} j_{b}}^{k_{d}}+\Gamma_{i_{a} j_{b+1}}^{k_{d}}-\Gamma_{i_{a} j_{b}}^{k_{d-1}}=\Gamma_{j_{b} i_{a+1}}^{k_{d}}-\Gamma_{j_{b} i_{a}}^{k_{d-1}} .
$$

Let

$$
\left(\begin{array}{ll}
B_{i, k, 0, j_{b}}=\Gamma_{i_{1} j_{b}}^{k_{1}}+\Gamma_{i_{2} j_{b}}^{k_{2}}+\cdots+\Gamma_{i_{y_{0}} j_{b}}^{k_{y_{0}}}, & y_{0}=\min \left\{\alpha_{i}, \alpha_{k}\right\}, \\
B_{i, k, 1, j_{b}}=\Gamma_{i_{2} j_{b}}^{k_{1}}+\Gamma_{i_{3} j_{b}}^{k_{2}}+\cdots+\Gamma_{i_{y_{1}} j_{b}}^{k_{y_{1}-1}}, & y_{1}=\min \left\{\alpha_{i}, \alpha_{k}+1\right\} \\
B_{i, k, 0, j_{b}}=\Gamma_{i_{3} j_{b}}^{k_{1}}+\Gamma_{i_{4} j_{b}}^{k_{2}}+\cdots+\Gamma_{i_{y_{2}} j_{b}}^{k_{y_{2}-2}}, & y_{2}=\min \left\{\alpha_{i}, \alpha_{k}+2\right\} \\
\vdots & \\
B_{i, k, \alpha_{i}-1, j_{b}}=\Gamma_{i_{\alpha_{i}} j_{b}}^{k_{1}}, &
\end{array}\right.
$$

with $\lambda_{j}=-(n / 2) \varepsilon H, \lambda_{i}=\lambda_{k} \neq-(n / 2) \varepsilon H$, and $i \neq k$. It follows from (53) that for $1 \leq b \leq \alpha_{j}$ and $0 \leq e \leq \alpha_{i}-2$,

$$
\left(\begin{array}{l}
\left(-\frac{n}{2} \varepsilon H-\lambda_{k}\right) B_{i, k, e, j_{b}}+B_{i, k, e, j_{b+1}}-B_{i, k, e+1, j_{b}}=0 \\
\left(-\frac{n}{2} \varepsilon H-\lambda_{k}\right) B_{i, k, \alpha_{i}-1, j_{b}}+B_{i, k, \alpha_{i}-1, j_{b+1}}=0
\end{array}\right.
$$

which implies that for $\lambda_{j}=-(n / 2) \varepsilon H, \lambda_{i}=\lambda_{k} \neq-(n / 2) \varepsilon H$, and $i \neq k$,

$$
B_{i, k, e, j_{b}}=0, \quad 1 \leq b \leq \alpha_{j}, 0 \leq e \leq \alpha_{i}-1
$$

Now, we treat the two cases that $\nabla H$ is not light-like or light-like separately and get contradictions.

Case 1. $\nabla H$ is not light-like.

As $\nabla H$ is in the direction of $u_{1_{\alpha_{1}}}, \nabla H$ is not light-like means that $\alpha_{1}=1$. Observe equation (38); we find if $\lambda_{i}=$ $-(n / 2) \varepsilon H$, then $u_{1_{1}}(H)=0$, which contradicts with (19). So, we conclude that $\lambda_{i} \neq-(n / 2) \varepsilon H$, for $2 \leq i \leq m$. Since there are two distinct values among $\left\{\lambda_{1}, \cdots, \lambda_{m}\right\}$ and $\operatorname{tr} A=n \varepsilon H$, we have $\lambda_{2}=\cdots=\lambda_{m}=3 n \varepsilon H / 2(n-1)$. It follows from (34) and (38) that

$$
\Gamma_{i_{a} 1_{1}}^{i_{a}}=-\frac{3 u_{1_{1}}(H)}{(n+2) H}, \quad 2 \leq i \leq m, 1 \leq a \leq \alpha_{i} .
$$

Denote $W=\Gamma_{i_{a} 1_{1}}^{i_{a}}$, we have the following lemmas. 
Lemma 2. We have

$$
u_{1_{1}}(W)+W^{2}=\frac{3 \varepsilon_{1} n^{2} H^{2}}{4(n-1)}-c \varepsilon_{1} .
$$

Proof. Since $\lambda_{i}=3 n \varepsilon H / 2(n-1)$ with $2 \leq i \leq m$, it follows from (46) that

$$
\left(-\frac{n}{2} \varepsilon H-\lambda_{j}\right) \Gamma_{i_{a} 1_{1}}^{j_{1}}=\Gamma_{1_{1} i_{a+1}}^{j_{1}}, \quad 2 \leq i, j \leq m, i \neq j,
$$

combining (43), we have

$$
\Gamma_{1_{1} i_{a+1}}^{j_{1}}=0
$$

with $2 \leq i, j \leq m, i \neq j$, and $2 \leq a \leq \alpha_{i}-1$.

Using Gauss equation for $\left\langle R\left(u_{1_{1}}, u_{i_{\alpha_{i}}}\right) u_{1_{1}}, u_{i_{1}}\right\rangle$, with $2 \leq i$ $\leq m$, combining (29), (31), (35), (40), (42), (43) and (60), we have

$$
\begin{aligned}
u_{1_{1}}\left(\Gamma_{i_{\alpha_{i}} 1_{1}}^{i_{\alpha_{i}}}\right)= & -\left(\Gamma_{i_{\alpha_{i}} 1_{1}}^{i_{\alpha_{i}}}\right)^{2}-\sum_{2 \leq j \leq m, j \neq i} \\
& \cdot\left(\Gamma_{i_{\alpha_{i}} 1_{1}}^{j_{\alpha_{j}}} \Gamma_{1_{1} j_{\alpha_{j}}}^{i_{\alpha_{i}}}-\Gamma_{j_{\alpha_{j}} 1_{1}}^{i_{\alpha_{i}}} \Gamma_{1_{1} i_{\alpha_{i}}}^{j_{\alpha_{\alpha_{j}}}}-\Gamma_{j_{\alpha_{j}-1} 1_{1}}^{i_{\alpha_{i}}} \Gamma_{1_{1} i_{\alpha_{i}}}^{j_{\alpha_{j}-1}}+\Gamma_{i_{\alpha_{i}} 1_{1}}^{j_{\alpha_{j}}} \Gamma_{j_{\alpha_{j}} 1_{1}}^{i_{\alpha_{i}}}\right) \\
& +\frac{3 \varepsilon_{1} n^{2} H^{2}}{4(n-1)}-c \varepsilon_{1} .
\end{aligned}
$$

From (22) and (23), we know $\Gamma_{i_{\alpha_{i}} 1_{1}}^{j_{\alpha_{j}}}=\varepsilon_{i} \varepsilon_{j} \Gamma_{j_{1} 1_{1}}^{i_{1}}, \Gamma_{j_{\alpha_{j}-1} 1_{1}}^{i_{\alpha_{i}}}=$ $\varepsilon_{i} \varepsilon_{j} \Gamma_{i_{1} 1_{1}}^{j_{2}}$, with $2 \leq i, j \leq m$. So, we can rewrite the above equation as

$$
\begin{aligned}
u_{1_{1}}\left(\Gamma_{i_{\alpha_{i}} 1_{1}}^{i_{\alpha_{i}}}\right)= & -\left(\Gamma_{i_{\alpha_{i}} 1_{1}}^{i_{\alpha_{i}}}\right)^{2}-\sum_{2 \leq j \leq m, j \neq i} \\
& \cdot\left(\Gamma_{i_{1} 1_{1}}^{j_{1}} \Gamma_{1_{1} j_{1}}^{i_{1}}+\Gamma_{i_{1} 1_{1}}^{j_{2}} \Gamma_{1_{1} j_{2}}^{i_{1}}-\Gamma_{j_{1} 1_{1}}^{i_{1}} \Gamma_{1_{1} i_{1}}^{j_{1}}+\Gamma_{i_{1} 1_{1}}^{j_{1}} \Gamma_{j_{1} 1_{1}}^{i_{1}}\right) \\
& +\frac{3 \varepsilon_{1} n^{2} H^{2}}{4(n-1)}-c \varepsilon_{1}, \quad 2 \leq i \leq m .
\end{aligned}
$$

For $2 \leq i, j \leq m$ and $i \neq j$, if $\alpha_{j}<\alpha_{i}$, then we have $\Gamma_{j_{1} 1_{1}}^{i_{1}}=$ $\Gamma_{j_{\alpha_{j}} 1_{1}}^{i_{\alpha_{j}}}=0$ from (22), (43), and (44). And if $\alpha_{j}=\alpha_{i}$, from (56), we know

$$
\Gamma_{j_{1} 1_{1}}^{i_{1}}+\Gamma_{j_{2} 1_{1}}^{i_{2}}+\cdots+\Gamma_{j_{\alpha_{j}} 1_{1}}^{i_{\alpha_{i}}}=0
$$

which together with (44), tells us that

$$
\Gamma_{j_{1} 1_{1}}^{i_{1}}=0
$$

So, for $2 \leq i, j \leq m$ and $i \neq j$, if $\alpha_{j} \leq \alpha_{i}$, then

$$
\Gamma_{j_{1} 1_{1}}^{i_{1}}=0
$$

For $2 \leq i, j \leq m$ and $i \neq j$, if $\alpha_{j}>\alpha_{i}$, combining (65), we get from (46) that

$$
\left\{\begin{array}{l}
-\frac{n(n+2) \varepsilon H}{2(n-1)} \Gamma_{i_{1} 1_{1}}^{j_{2}}=\Gamma_{1_{1} i_{2}}^{j_{2}}-\Gamma_{1_{1} i_{1}}^{j_{1}}, \\
-\frac{n(n+2) \varepsilon H}{2(n-1)} \Gamma_{i_{2} 1_{1}}^{j_{3}}=\Gamma_{1_{1} i_{3}}^{j_{3}}-\Gamma_{1_{1} i_{2}}^{j_{2}}, \\
\vdots \\
-\frac{n(n+2) \varepsilon H}{2(n-1)} \Gamma_{i_{\alpha_{i}-1} 1_{1}}^{j_{\alpha_{i}}}=\Gamma_{1_{1} i_{\alpha_{i}}}^{j_{\alpha_{i}}}-\Gamma_{1_{1} i_{\alpha_{i}-1}}^{j_{\alpha_{i}-1}}, \\
-\frac{n(n+2) \varepsilon H}{2(n-1)} \Gamma_{i_{\alpha_{i}} i_{1}}^{j_{\alpha_{i}+1}}=-\Gamma_{1_{1} i_{\alpha_{i}}}^{j_{\alpha_{i}}} .
\end{array}\right.
$$

If $\alpha_{j}>\alpha_{i}+1$, then (43) and (44) give us that

$$
\Gamma_{i_{1} 1_{1}}^{j_{2}}=\cdots=\Gamma_{i_{\alpha_{i}} 1_{1}}^{j_{\alpha_{i}+1}}=0 .
$$

As (67), it follows from (66) that for $2 \leq i, j \leq m$ and $i \neq j$, if $\alpha_{j}>\alpha_{i}+1$, then

$$
\Gamma_{1_{1} i_{1}}^{j_{1}}=0
$$

For $2 \leq i, j \leq m$ and $i \neq j$, if $\alpha_{j}=\alpha_{i}+1$, then combining (22) and (23), we get from (44) and (66) that

$$
\begin{aligned}
& \Gamma_{i_{1} 1_{\alpha_{1}}}^{j_{2}}=\Gamma_{i_{\alpha_{i}} 1_{\alpha_{1}}}^{j_{\alpha_{j}}}=\varepsilon_{i} \varepsilon_{j} \Gamma_{j_{1} 1_{\alpha_{1}}}^{i_{1}}, \\
& \Gamma_{1_{\alpha_{1}} i_{1}}^{j_{1}}=\alpha_{i} \Gamma_{1_{\alpha_{1}} i_{\alpha_{i}}}^{j_{\alpha_{\alpha_{1}}}}=\varepsilon_{i} \varepsilon_{j} \frac{n(n+2) \varepsilon H}{2(n-1)} \alpha_{i} \Gamma_{j_{1} 1_{\alpha_{1}}}^{i_{1}} .
\end{aligned}
$$

Therefore, from (59), (65), (67), (68), and (69), (62) can be simplified to

$$
\begin{aligned}
& \frac{n(n+2) \varepsilon H}{2(n-1)} \varepsilon_{i}\left\{\sum_{j=2, \alpha_{j}=\alpha_{i}-1}^{m} \varepsilon_{j} \alpha_{j}\left(\Gamma_{i_{1} 1_{1}}^{j_{1}}\right)^{2}-\sum_{j=2, \alpha_{j}=\alpha_{i}+1}^{m} \varepsilon_{j}\left(\alpha_{i}+1\right)\left(\Gamma_{j_{1} 1_{1}}^{i_{1}}\right)^{2}\right\} \\
& \quad+u_{1_{1}}\left(\Gamma_{i_{1} 1_{1}}^{i_{1}}\right)+\left(\Gamma_{i_{1} 1_{1}}^{i_{1}}\right)^{2}=\frac{3 \varepsilon_{1} n^{2} H^{2}}{4(n-1)}-c \varepsilon_{1}, \quad 2 \leq i \leq m .
\end{aligned}
$$

Choosing $\left\{\alpha_{1_{1}}, \alpha_{1_{2}}, \cdots, \alpha_{1_{\xi_{1}}}, \alpha_{2_{1}}, \alpha_{2_{2}}, \cdots, \alpha_{\xi_{\xi_{2}}}, \cdots, \alpha_{h_{1}}, \alpha_{h_{2}}, \cdots\right.$, $\left.\alpha_{h_{\xi_{h}}}\right\}$ in $\left\{\alpha_{2}, \cdots, \alpha_{m}\right\}$, such that

$$
\tilde{\alpha}_{i}:=\alpha_{i_{1}}=\alpha_{i_{2}}=\cdots=\alpha_{i_{\xi_{i}}}, \tilde{\alpha}_{i}+1=\tilde{\alpha}_{i+1}, \quad 1 \leq i \leq h,
$$

and $\tilde{\alpha}_{1}-1, \tilde{\alpha}_{h}+1 \notin\left\{\alpha_{2}, \cdots, \alpha_{m}\right\}$. 
Taking sum in (70) for $i=k_{1}, k_{2}, \cdots, k_{\xi_{k}}$, with $1 \leq k \leq h$, we have

$$
\left(\begin{array}{l}
-\left(\tilde{\alpha}_{1}+1\right) Q_{1}=\xi_{1} U, \\
\tilde{\alpha}_{1} Q_{1}-\left(\tilde{\alpha}_{2}+1\right) Q_{2}=\xi_{2} U, \\
\tilde{\alpha}_{2} Q_{2}-\left(\tilde{\alpha}_{3}+1\right) Q_{3}=\xi_{3} U, \\
\vdots \\
\tilde{\alpha}_{h-2} Q_{h-2}-\left(\tilde{\alpha}_{h-1}+1\right) Q_{h-1}=\xi_{h-1} U, \\
\tilde{\alpha}_{h-1} Q_{h-1}=\xi_{h} U
\end{array}\right.
$$

where

$$
\begin{aligned}
U & =-u_{1_{1}}(W)-W^{2}+\frac{3 \varepsilon_{1} n^{2} H^{2}}{4(n-1)}-c \varepsilon_{1}, \\
Q_{k} & =\frac{n(n+2) \varepsilon H}{2(n-1)} \sum_{\substack{1 \leq p \leq \xi_{k} \\
1 \leq q \leq \xi_{k+1}}} \varepsilon_{\rho} \varepsilon_{\sigma}\left(\Gamma_{\sigma_{1} 1_{\alpha_{1}}}^{\rho_{1}}\right)^{2}, \quad \rho=k_{p}, \sigma=(k+1)_{q} .
\end{aligned}
$$

From (72), we can get $Q_{k}=0,1 \leq k \leq h-1$, and $U=0$, i.e., (58) holds.

Lemma 3. We have

$\sum_{i=2}^{m} \sum_{a=1}^{\alpha_{i}} \Gamma_{i_{a} 1_{1}}^{i_{a}} u_{1_{1}}(H)+u_{1_{1}} u_{1_{1}}(H)-\varepsilon_{1} \varepsilon \frac{(n+8) n^{2} H^{3}}{4(n-1)}+n c \varepsilon_{1} H=0$.

Proof. For $1 \leq k \leq m$, if $\alpha_{k}$ is an even number, we put

$$
e_{k_{a}}=\frac{u_{k_{a}}-u_{k_{\alpha_{k}-a+1}}}{\sqrt{2}}, k_{\alpha_{k}-a+1}=\frac{u_{k_{a}}+u_{k_{\alpha_{k}-a+1}}}{\sqrt{2}},
$$

with $1 \leq a \leq \alpha_{k} / 2$. And if $\alpha_{k}$ is an odd number, we take

$$
e_{k_{a}}=\frac{u_{k_{a}}-u_{k_{\alpha_{k}-a+1}}}{\sqrt{2}}, k_{\alpha_{k}-a+1}=\frac{u_{k_{a}}+u_{k_{\alpha_{k}-a+1}}}{\sqrt{2}}, \quad 1 \leq a<\frac{\alpha_{k}+1}{2},
$$

and $e_{k_{\left(\alpha_{k}+1\right) / 2}}=u_{k_{\left(\alpha_{k}+1\right) / 2}}$. We easily find $\mathfrak{E}_{k}=\left\{e_{k_{1}}, e_{k_{2}}, \cdots, e_{k_{\alpha_{k}}}\right\}$ is an orthonormal basis of $V_{k}$ with $1 \leq k \leq m$. Thus, $\mathfrak{E}=$ $\left\{e_{k_{a}}, 1 \leq k \leq m, 1 \leq a \leq \alpha_{k}\right\}$ is an orthonormal basis of $T_{x} M_{r}^{n}$.
From (19), (75), and (76), we have when $\alpha_{k}$ is an even number,

$$
\begin{aligned}
& \nabla_{e_{k_{a}}} e_{k_{a}}(H) \\
& \quad=\frac{1}{2}\left(\Gamma_{k_{a} k_{a}}^{1}+\Gamma_{k_{\alpha_{k}-a+1} k_{\alpha_{k}-a+1}}^{1_{1}}-\Gamma_{k_{a} k_{\alpha_{k}-a+1}}^{1_{1}}-\Gamma_{k_{\alpha_{k}-a+1} k_{a}}^{1_{1}}\right) u_{1_{1}}(H), \\
& \nabla_{e_{k_{\alpha_{k}-a+1}}} e_{k_{\alpha_{k}-a+1}}(H) \\
& \quad=\frac{1}{2}\left(\Gamma_{k_{a} k_{a}}^{1_{1}}+\Gamma_{k_{\alpha_{k}-a+1} k_{\alpha_{k}-a+1}}^{1_{1}}+\Gamma_{k_{a} k_{\alpha_{k}-a+1}}^{1_{1}}+\Gamma_{k_{\alpha_{k}-a+1} k_{a}}^{1_{1}}\right) u_{1_{1}}(H),
\end{aligned}
$$

with $1 \leq \alpha \leq \alpha_{k} / 2$, and when $\alpha_{k}$ is an odd number,

$$
\begin{aligned}
& \nabla_{e_{k_{a}}} e_{k_{a}}(H) \\
& =\frac{1}{2}\left(\Gamma_{k_{a} k_{a}}^{1_{1}}+\Gamma_{k_{\alpha_{k}-a+1} k_{\alpha_{k}-a+1}}^{1_{1}}-\Gamma_{k_{a} k_{\alpha_{k}-a+1}}^{1_{1}}-\Gamma_{k_{\alpha_{k}-a+1} k_{a}}^{1_{1}}\right) u_{1_{1}}(H), \\
& \nabla_{e_{k_{\alpha_{k}-a+1}}} e_{k_{\alpha_{k}-a+1}}(H) \\
& =\frac{1}{2}\left(\Gamma_{k_{a} k_{a}}^{1_{1}}+\Gamma_{k_{\alpha_{k}-a+1} k_{\alpha_{k}-a+1}}^{1_{1}}+\Gamma_{k_{a} k_{\alpha_{k}-a+1}}^{1_{1}}+\Gamma_{k_{\alpha_{k}-a+1} k_{a}}^{1_{1}}\right) u_{1_{1}}(H), \\
& \nabla_{e_{\left(_{\left(\alpha_{k}+1\right) / 2}\right.}} e_{k_{\left(\alpha_{k}+1\right) / 2}}(H) \\
& =\Gamma_{k_{\left(\alpha_{k}+1\right) / 2} k_{\left(\alpha_{k}+1\right) / 2}}^{l_{1}} u_{1_{1}}(H) \text {, }
\end{aligned}
$$

with $1 \leq \alpha<\left(\alpha_{k}+1\right) / 2$

Note that $e_{1_{1}}=u_{1_{1}}$; it follows from (19) that

$$
e_{1_{1}}(H) \neq 0, e_{i_{a}}(H)=0, \quad 2 \leq i \leq m, 1 \leq a \leq \alpha_{i} .
$$

By calculating, we get

$$
\operatorname{tr} A^{2}=\frac{(n+8) n^{2} H^{2}}{40}
$$

Since the above, we obtain from (6) that

$$
\sum_{i=2}^{m} \sum_{a=1}^{\alpha_{i}} \Gamma_{i_{a} 1_{1}}^{i_{a}} u_{1_{1}}(H)+u_{1_{1}} u_{1_{1}}(H)-\varepsilon_{1} \varepsilon \frac{(n+8) n^{2} H^{3}}{4(n-1)}+n c \varepsilon_{1} H=0 .
$$

Now, we continue the proof of Theorem 1 for Case 1. Combining (57) and (58), we get

$$
\begin{aligned}
& u_{1_{1}} u_{1_{1}}(H)-\frac{(n+2)(n+5) H}{9} W^{2}-\frac{(n+2) n^{2} \varepsilon_{1} H^{3}}{4(n-1)} \\
& +\frac{1}{3}(n+2) c \varepsilon_{1} H .
\end{aligned}
$$


Substitute the above equation into (74), considering $\alpha_{2}+\cdots+\alpha_{m}=n\left(\alpha_{1}=1\right)$ and (57), we have

$\left\{\frac{2(n-4)(n+2)}{9} W^{2}+\frac{(n+2+\varepsilon(n+8)) n^{2} \varepsilon_{1} H^{2}}{4(n-1)}-\frac{2}{3}(2 n+1) c \varepsilon_{1}\right\} H=0$.

Act on (83) with $u_{1_{1}}$, and using (57) and (58), we obtain

$\left\{\frac{2(n-4)(n+2)}{9} W^{2}+\frac{(n+2+\varepsilon(n+8)) n^{2} \varepsilon_{1} H^{2}}{4(n-1)}-\frac{2}{3}(2 n+1) c \varepsilon_{1}\right\} u_{1_{1}}$

$(H)-2(n+2)$

$\left\{\frac{2(n-4)}{9} W^{2}+\frac{(n-10-\varepsilon(n+8)) n^{2} \varepsilon_{1} H^{2}}{12(n-1)}+\frac{2(n-4)}{9} c \varepsilon_{1}\right\} H W=0$.

As (83) and $W \neq 0$, the above equation implies that

$\left\{\frac{2(n-4)}{9} W^{2}+\frac{(n-10-\varepsilon(n+8)) n^{2} \varepsilon_{1} H^{2}}{12(n-1)}+\frac{2(n-4)}{9} c \varepsilon_{1}\right\} H=0$,

which together with (83) gives that

$$
\begin{aligned}
& \frac{[-(n+2)(n-13)+(n+8)(n+5) \varepsilon] n^{2} \varepsilon_{1} H^{3}}{12(n-1)} \\
& \quad-\frac{2}{9}(n-1)(n+5) c \varepsilon_{1} H=0,
\end{aligned}
$$

which implies that $H$ is a constant, a contradiction.

Case 2. $\nabla H$ is light-like.

Notice that $\nabla H$ is light-like means $\alpha_{1} \geq 2$. Since $\operatorname{tr} A=n$ $\varepsilon H$, we can suppose $\lambda_{1}=\cdots=\lambda_{p}=-(n / 2) \varepsilon H$ and $\lambda_{p+1}=\cdots$ $=\lambda_{m}=\left(2+\alpha_{1}+\cdots+\alpha_{p}\right) n \varepsilon H /\left(2+\alpha_{p+1}+\cdots+\alpha_{m}\right)$. It follows from (19) and (38) that

$$
\Gamma_{i_{1} 1 \alpha_{1}}^{i_{1}}=0, \quad p+1 \leq i \leq m
$$

Calculate $\left\langle R\left(u_{1_{2}}, u_{i_{a}}\right) u_{1_{\alpha_{1}}}, u_{i_{\alpha_{i}-a+1}}\right\rangle$ and $\left\langle R\left(u_{1_{1}}, u_{i_{a}}\right) u_{1_{\alpha_{1}}}\right.$, $\left.u_{i_{\alpha_{i}-a+1}}\right\rangle$ with $p+1 \leq i \leq m$ by Gauss equation, and letting $i$ and $a$ for sum with $p+1 \leq i \leq m$ and $1 \leq a \leq \alpha_{i}$, combining (21), (22), (23) (26), (28), (29), (35), (42), (45), and (87), we get

$$
\begin{aligned}
& \sum_{i=p+1}^{m} \sum_{a=1}^{\alpha_{i}}\left\{\sum_{d=3}^{\alpha_{1}-1} \Gamma_{1_{2} 1 \alpha_{1}}^{1_{d}} \Gamma_{i_{a} 1_{d}}^{i_{a}}+\sum_{k=2}^{p} \sum_{d=2}^{\alpha_{k}} \Gamma_{1_{2} 1 \alpha_{1}}^{k_{d}} \Gamma_{i_{a} k_{d}}^{i_{a}}\right\} \\
& -\sum_{i=p+1}^{m} \sum_{1 \leq b<a \leq \alpha_{i}} \Gamma_{i_{a} 1_{2}}^{i_{b}} \Gamma_{i_{b} 1_{\alpha_{1}}}^{i_{a}}-\sum_{\substack{p+1 \leq k \leq m \\
k \neq i}} \sum_{\substack { a=1 \\
\begin{subarray}{c}{1 \leq b \leq \alpha_{k} \\
b<a{ a = 1 \\
\begin{subarray} { c } { 1 \leq b \leq \alpha _ { k } \\
b < a } } \end{subarray} \Gamma_{i_{a} 1_{2}}^{k_{b}} \Gamma_{k_{b} 1_{\alpha_{1}}}^{i_{a}}=0,}^{\alpha_{i}}=0,
\end{aligned}
$$

$$
\begin{aligned}
& \sum_{i=p+1}^{m} \sum_{a=1}^{\alpha_{i}}\left\{\sum_{d=2}^{\alpha_{1}-1} \Gamma_{1_{1} 1 \alpha_{1}}^{1_{1}} \Gamma_{i_{a} 1_{d}}^{i_{a}}+\sum_{k=2}^{p} \sum_{d=1}^{\alpha_{k}} \Gamma_{1_{2} 1 \alpha_{1}}^{k_{\alpha_{1}}} \Gamma_{i_{a} k_{d}}^{i_{a}}\right\} \\
& -\sum_{i=p+1}^{m} \sum_{1 \leq b<a \leq \alpha_{i}} \Gamma_{i_{a} 1_{1}}^{i_{b}} \Gamma_{i_{b} 1_{\alpha_{1}}}^{i_{a}}-\sum_{p+1 \leq k \leq m} \sum_{\substack{k \neq 1 \\
k \neq i}}^{\alpha_{i}} \sum_{\substack{\leq b \leq \alpha_{k} \\
b<a}} \Gamma_{i_{a} 1_{1}}^{k_{b}} \Gamma_{k_{b} 1_{\alpha_{1}}}^{i_{a}} \\
& =\left(\alpha_{p+1}+\cdots+\alpha_{m}\right)\left(c \varepsilon_{1}-\frac{1}{2} n \varepsilon_{1} H \lambda_{m}\right) .
\end{aligned}
$$

Since (50) and (56), we find

$$
\sum_{i=p+1}^{m} \sum_{1 \leq b<a \leq \alpha_{i}} \Gamma_{i_{a} 1_{d}}^{i_{b}} \Gamma_{i_{b} 1_{\alpha_{1}}}^{i_{a}}=\sum_{\substack{p+1 \leq k \leq m \\ k \neq i}} \sum_{\substack{a=1 \\ \alpha_{i}}} \sum_{\substack{1 \leq b \leq \alpha_{k} \\ b<a}} \Gamma_{i_{a} 1_{d}}^{k_{b}} \Gamma_{k_{b} 1_{\alpha_{1}}}^{i_{a}}=0 .
$$

So, (88) and (89) can be simplified to

$$
\begin{gathered}
\sum_{i=p+1}^{m} \sum_{a=1}^{\alpha_{i}}\left\{\sum_{d=3}^{\alpha_{1}-1} \Gamma_{1_{2} 1 \alpha_{1}}^{1_{d}} \Gamma_{i_{a} 1_{d}}^{i_{a}}+\sum_{k=2}^{p} \sum_{d=2}^{\alpha_{k}} \Gamma_{1_{2} 1 \alpha_{1}}^{k_{d}} \Gamma_{i_{a}}^{i_{a}} k_{d}\right\}=0, \\
\sum_{i=p+1}^{m} \sum_{a=1}^{\alpha_{i}}\left\{\sum_{d=2}^{\alpha_{1}-1} \Gamma_{1_{1} 1 \alpha_{1}}^{1_{d}} \Gamma_{i_{a} 1_{d}}^{i_{a}}+\sum_{k=2}^{p} \sum_{d=1}^{\alpha_{k}} \Gamma_{1_{1} 1 \alpha_{1}}^{k_{d}} \Gamma_{i_{a} k_{d}}^{i_{a}}\right\} \\
=\left(\alpha_{p+1}+\cdots+\alpha_{m}\right)\left(c \varepsilon_{1}-\frac{1}{2} n \varepsilon_{1} H \lambda_{m}\right) .
\end{gathered}
$$

It follows from (52) that

$$
\sum_{i=p+1}^{m} \sum_{a=1}^{\alpha_{i}}\left\{\left(-\frac{n}{2} \varepsilon H-\lambda_{m}\right) \Gamma_{i_{a} k_{d}}^{i_{a}}+\Gamma_{i_{a} k_{d+1}}^{i_{a}}\right\}=0
$$

with $1 \leq k \leq p$ and $k_{d} \neq 1_{1}$.

Multiply both sides of (92) with $-(n / 2) \varepsilon H-\lambda_{m}$, we get

$$
\begin{aligned}
& \sum_{d=2}^{\alpha_{1}-1} \Gamma_{1_{1} 1 \alpha_{1}}^{1_{d}} \sum_{i=p+1}^{m} \sum_{a=1}^{\alpha_{i}}\left(-\frac{n}{2} \varepsilon H-\lambda_{m}\right) \Gamma_{i_{a} 1_{d}}^{i_{a}} \\
& \quad+\sum_{k=2}^{p} \sum_{d=1}^{\alpha_{k}} \Gamma_{1_{a} 1 \alpha_{1}}^{k_{d}} \sum_{i=p+1}^{m} \sum_{a=1}^{\alpha_{i}}\left(-\frac{n}{2} \varepsilon H-\lambda_{m}\right) \Gamma_{i_{a} k_{d}}^{i_{a}} \\
& =\varepsilon_{1}\left(\alpha_{p+1}+\cdots+\alpha_{m}\right)\left(-\frac{n}{2} \varepsilon H-\lambda_{m}\right)\left(c-\frac{1}{2} n H \lambda_{m}\right) .
\end{aligned}
$$

Combining (87) and (93), it gives us

$$
\begin{gathered}
\sum_{d=2}^{\alpha_{1}-2} \Gamma_{1_{1} 1 \alpha_{1}}^{1_{1}} \sum_{i=p+1}^{m} \sum_{a=1}^{\alpha_{i}} \Gamma_{i_{a} 1_{d+1}}^{i_{a}}-\sum_{k=2}^{p} \sum_{d=1}^{\alpha_{k}-1} \Gamma_{1_{a} 1 \alpha_{1}}^{k_{d}} \sum_{i=p+1}^{m} \sum_{a=1}^{\alpha_{i}} \Gamma_{1_{a} k_{d+1}}^{k_{d}} \\
=\varepsilon_{1}\left(\alpha_{p+1}+\cdots+\alpha_{m}\right)\left(-\frac{n}{2} \varepsilon H-\lambda_{m}\right)\left(c-\frac{1}{2} n H \lambda_{m}\right) .
\end{gathered}
$$


Since (91), we get from (95) that

$$
\left(-\frac{n}{2} \varepsilon H-\lambda_{m}\right)\left(c-\frac{1}{2} n H \lambda_{m}\right)=0
$$

which combining $\lambda_{m} \neq-(n / 2) \varepsilon H$ and $\lambda_{m}=\left(2+\alpha_{1}+\cdots+\alpha_{p}\right)$ $n \varepsilon H / 2\left(\alpha_{p+1}+\cdots+\alpha_{m}\right)$, tells us that $c-\left(\left(2+\alpha_{1}+\cdots+\alpha_{p}\right) n^{2} \varepsilon H^{2}\right.$ $\left./ 4\left(\alpha_{p+1}+\cdots+\alpha_{m}\right)\right)=0$.

So, $H$ is a constant, a contradiction.

In view of the two cases, we complete the proof of Theorem 1 .

Remark 4. With the assumption that the shape operator is diagonalizable, the result of Theorem 1 was proved in $[6,8$, 12] when the hypersurface $M_{r}^{n}$ has at most three distinct principal curvatures.

Applying Theorem 1, we can get the following theorem.

Theorem 5. Let $M_{r}^{n}$ be a nondegenerate biharmonic hypersurface of pseudo-Riemannian space form $N_{s}^{n+1}(c)$, with $c \varepsilon \leq 0$. Suppose that $M_{r}^{n}$ has at most two distinct principal curvatures, which are all real numbers, then $M_{r}^{n}$ is minimal.

Proof. We know from Theorem 1 that $H$ is a constant; it follows from (6) that

$$
H \operatorname{tr} A^{2}=n c \varepsilon H
$$

When $c \varepsilon \leq 0$, it is easy to see from (97) that $H=0$. When $c \varepsilon \leq 0$, then (97) implies $H=0$, or $\operatorname{tr} A^{2}=0$. Since $\operatorname{tr} A^{2}$ is equal to the sum of the squares of all principal curvatures and $\operatorname{tr} A^{2}$ is equal to the sum of all principal curvatures, so $\operatorname{tr} A^{2}=0$ tells us $t r A^{2}=0$. Combining $\operatorname{tr} A=n \varepsilon H$, we have $H$ $=0$, i.e., $M_{r}^{n}$ is minimal.

For odd-dimensional hypersurfaces, under the assumption that hypersurfaces have at most two distinct principal curvatures, the principal curvatures are all real. So, from Theorem 5 , the following theorems are true.

Theorem 6. Let $M_{r}^{2 k-1}$ be a nondegenerate biharmonic hypersurface of pseudo-Euclidean space $\mathbb{E}_{s}^{2 k}$. Suppose that $M_{r}^{2 k-1}$ has at most two distinct principal curvatures, then $M_{r}^{2 k-1}$ is minimal.

Theorem 7. Let $M_{s-1}^{2 k-1}$ be a nondegenerate biharmonic hypersurface of de Sitter space $\mathbb{S}_{s}^{2 k}(c)$. Suppose that $M_{s-1}^{2 k-1}$ has at most two distinct principal curvatures, then $M_{s-1}^{2 k-1}$ is minimal.

Theorem 8. Let $M_{s}^{2 k-1}$ be a nondegenerate biharmonic hypersurface of anti-de Sitter space $\mathbb{H}_{s}^{2 k}(c)$. Suppose that $M_{s}^{2 k-1}$ has at most two distinct principal curvatures, then $M_{s}^{2 k-1}$ is minimal.

Remark 9. Under the assumption that the shape operator is diagonalizable, the results of Theorems 6,7 , and 8 were proven not only for odd-dimensional hypersurfaces but also for even-dimensional hypersurfaces (cf. $[6,8]$ ).

\section{Data Availability}

The data used to support the findings of this study are included within the article.

\section{Conflicts of Interest}

The authors declare that they have no conflicts of interest.

\section{Acknowledgments}

This work was supported by the NSF of China (11761061), the University Project of Gansu Province (2019B-045), and the Project of Northwest Normal University (NWNULKQN2019-23).

\section{References}

[1] A. Arvanitoyeorgos, F. Defever, G. Kaimakamis, and V. J. Papantoniou, "Biharmonic Lorentz hypersurfaces in $E_{1}^{4}$," Pacific Journal of Mathematics, vol. 229, no. 2, article 293C305, pp. 293-305, 2007.

[2] B. Y. Chen and S. Ishikawa, "Biharmonic surfaces in pseudoEuclidean spaces," Memoirs of the Faculty of Science, Kyushu University. Series A, Mathematics, vol. 45, no. 2, pp. 323-347, 1991.

[3] B. Y. Chen and S. Ishikawa, "Biharmonic pseudo-Riemannian submanifolds in pseudo-Euclidean spaces," Kyushu Journal of Mathematics, vol. 52, no. 1, pp. 167-185, 1998.

[4] B. Y. Chen, Total mean curvature and submanifolds of finite type (second edition), vol. 27 of Series in Pure Mathematics, World Scientific, Singapore, 2014.

[5] F. Defever, G. Kaimakamis, and V. Papantoniou, "Biharmonic hypersurfaces of the 4-dimensional semi-Euclidean space $\mathbb{E}_{s}^{4}$," Journal of Mathematical Analysis and Applications, vol. 315, no. 1, pp. 276-286, 2006.

[6] Y.-X. Dong and Y.-L. Ou, "Biharmonic submanifolds of pseudo-Riemannian manifolds," Journal of Geometry and Physics, vol. 112, pp. 252-262, 2017.

[7] J.-C. Liu and C. Yang, "Hypersurfaces in $\mathbb{E}_{s}^{n+1}$ satisfying $\Delta \vec{H}$ $=\lambda \vec{H}$ with at most three distinct principal curvatures," Journal of Mathematical Analysis and Applications, vol. 419, no. 1, pp. 562-573, 2014.

[8] J.-C. Liu and L. Du, "Classification of proper biharmonic hypersurfaces in pseudo-Riemannian space forms," Differential Geometry and its Applications, vol. 41, pp. 110-122, 2015.

[9] B. O'Neill, Semi-Riemannian Geometry: With Applications to Relativity, Pure and Applied Mathematics 103, Academic Press, NY, USA, 1983.

[10] V. J. Papantoniou and K. Petoumenos, "Biharmonic hypersurfaces of type $M_{2}^{3}$ in $\mathbb{E}_{2}^{4}$," Houston Journal of Mathematics, vol. 38, no. 1, pp. 93-114, 2012.

[11] T. Sasahara, "Biharmonic submanifolds in nonflat Lorentz 3space forms," Bulletin of the Australian Mathematical Society, vol. 85, no. 3, pp. 422-432, 2012.

[12] D. Yang and Y. Fu, "Biharmonic hypersurfaces in pseudoRiemannian space forms," International Journal of Geometric Methods in Modern Physics, vol. 13, no. 7, article 1650094, 2016. 